\title{
Computer-Aided Evaluation of Blood Vessel Geometry From Acoustic Images
}

Stefan B Lindström, Fredrik Uhlin, Niclas Bjarnegård, Micael Gylling, Kamilla Nilsson, Christina Svensson, Pia Yngman-Uhlin and Toste Länne

The self-archived postprint version of this journal article is available at Linköping University Institutional Repository (DiVA):

http:/ / urn.kb.se/ resolve?urn=urn:nbn:se:liu:diva- 144016

N.B.: When citing this work, cite the original publication.

Lindström, S. B, Uhlin, F., Bjarnegård, N., Gylling, M., Nilsson, K., Svensson, C., Yngman-Uhlin, P., Länne, T., (2017), Computer-Aided Evaluation of Blood Vessel Geometry From Acoustic Images, J ournal of ultrasound in medicine. https:// doi.org/ 10.1002/jum.14439

Original publication available at:

https:// doi.org/ 10.1002/jum.14439

Copyright: Wiley (12 months)

http:// eu.wiley.com/WileyCDA/ 


\section{Computer-aided evaluation of blood vessel geometry from}

2

4

5

6

7

8

9

10

11

12

13

$14 *$ Corresponding author. mail: IEI, Linkoping University, Sweden; email:

15 stefan.lindtroem@gmail.com; telephone: +46 13 281127; fax: +46 13149403

\section{acoustic images}

\author{
Running title: Computer-aided evaluation of blood vessel geometry
}

Stefan B. Lindström PhD ${ }^{\mathrm{a} *}$, Fredrik Uhlin RN, $\mathrm{PhD}^{\mathrm{b}, \mathrm{c}}$, Niclas Bjarnegård BMS, $\mathrm{PhD}^{\mathrm{b}}$, Micael Gylling RN ${ }^{\mathrm{b}}$, Kamilla Nilsson BMS ${ }^{\mathrm{b}}$, Christina Svensson $\mathrm{BMS}^{\mathrm{b}}$, Pia Yngman-Uhlin RN, $\mathrm{PhD}^{\mathrm{b}, \mathrm{d}}$, and Toste Länne $\mathrm{MD}, \mathrm{PhD}^{\mathrm{b}}$

${ }^{\mathrm{a}}$ Department of Management and Engineering, Linköping University, Linköping, Sweden

${ }^{\mathrm{b}}$ Department of Medical and Health Sciences, Linköping University, Linköping, Sweden

'Department of Biomedical Engineering, Technomedicum, Tallinn University of Technology, Tallinn, Estonia

${ }^{\mathrm{d}}$ Research and Development Unit in Local Health Care, Linköping University, Linköping, Sweden 


\section{Summary}

18 A method for computer-aided assessment of blood vessel geometries based on shape-fitting

19 algorithms from metric vision is evaluated. Acoustic images of cross-sections of the radial

20 artery and the cephalic vein were acquired, and medical practitioners used a computer

21 application to measure the wall thickness and nominal diameter of these blood vessels with a

22 caliper method and the shape-fitting method. The two methods perform equally well for wall

23 thickness measurements. The shape-fitting method is preferable for measuring the diameter,

24 since it reduces systematic errors by up to $63 \%$ in the case of the cephalic vein due to its

25 eccentricity.

26 Keywords — shape-fitting; lumen diameter; blood vessel wall; computer-aided assessment 


\section{Introduction}

29 With high frequency ultrasound, images of the human blood vessels can be obtained with high resolution, which makes it a feasible method in evaluation of luminal size and wall thickness. In clinical practice, distance and area measurements are done on frozen B-mode ultrasound images by manual placement of two calipers and fitting a line between multiple points. The accuracy and precision of such measurements need to be high in order to identify true changes of wall thickness and lumen diameter in subjects over time, caused by either normal physiological adaptation or by unfavorable pathophysiological mechanisms that leads to vascular remodeling. Several theoretical models describing the adaptation of blood vessels have been proposed in recent years ${ }^{1-4}$, but without in vivo measurements of the evolution of the blood vessel geometry, there is little hope of properly validating the models or achieving the predictive capability needed to employ them for, e.g., planning treatments or surgical 40 procedures.

41 The turnover of the main constituents of blood vessels - collagen and vascular smooth muscle ${ }^{5}$-is approximately 80 days $^{6}$, which reflects the time-scale of adaptation. To observe such adaptation, it is necessary to make repeated measurements of the blood vessel geometry over several months. Ultrasonic imaging, being a relatively low-cost, highly accessible, noninvasive technique, is therefore a feasible option for conducting such measurements. In this work, we consider two types of blood vessel cross-section images: a transverse section is a section through the polar-radial plane, and a longitudinal section is a section through the 48 longitudinal-radial plane of the vessel.

49 Consider the acoustic image in Fig. 1a of a transverse section of a radial artery imaged 2 to $4 \mathrm{~cm}$ from the wrist. The imaged cross-section appears elliptical rather than circular, which may be due to the following reasons: the image plane is not perfectly perpendicular to the 
longitudinal axis of the vessel, the pressure of the acoustic probe against the skin deforms the vessel, or the cross-section of the vessel has an elliptic equilibrium shape. These three effects all lead to a finite eccentricity of the blood vessel's cross-section (Fig. 1h). Since the diameter of an ellipse varies with its orientation, it is not straight-forward to evaluate the diameter of the vessel using point-to-point distance measurements. Another difficulty is the poor acoustic contrast between the vessel wall and the surrounding tissue and blood, which makes it difficult to identify the interfaces of the blood vessel wall (Fig. 1a). This impairs the accuracy of the measurements of wall thickness and diameter. Similar problems arise for the

60 longitudinal section of the vessel (Fig. 2a).

In the field of metric vision, the geometries of objects are quantified from an image. Preprocessing of the image yields point sets of interest, typically outlined edges, to which geometric shapes such as parallel lines, circles or ellipses are fit ${ }^{7,8}$. Such methods are used in, e.g., automotive driver assistance ${ }^{9-11}$, industrial automation/inspection ${ }^{11,12}$, and eye-tracking applications ${ }^{13}$ where the circular iris and pupil appears as ellipses due to perspective projection. However, completely automated metric vision methods are difficult to apply to acoustic images of blood vessels due to their high noise level. No distinct edges can be observed at the pixel level of scale (Fig. 1a and Fig. 2a). Therefore, we propose a hybrid method where medical professionals outline the edges of the blood vessel wall using a graphical user interface (GUI). A computer program then fits geometric shapes to these point sets using the methods developed for metric vision.

In this work, we describe a new method for computer-aided evaluation of the cross-section geometry of blood vessels in terms of the vessel wall thickness and the nominal diameter. In the case of transverse sections, we choose to fit concentric ellipses; these geometric objects only have six degrees of freedom, and thus require very few points of interest to be properly 
defined, with better averaging achieved for a greater number of supplied points. For the same reasons, we choose polynomials for fitting the wall boundaries of the longitudinal sections. Each measurement yields the blood vessel wall thickness $h$, defined as the total thickness of the intima and media, and the nominal diameter $d$, defined as the circumference of the midplane of the blood vessel divided by $\pi$. We evaluate how the measured dimensions, $h$ and $d$, differ for caliper measurements and shape-fitting measurements in the cases of transverse and longitudinal sections, respectively. As a result, we identify the tendency for different measurement techniques to produce systematic errors.

\section{Materials and Methods}

Six presumably healthy individuals, three women and three men, in the age range of 28 to 58 years participated in the study. The radial artery (RA) and the cephalic vein (CV) were imaged at a distance between 2 and $4 \mathrm{~cm}$ from the wrist using high frequency $(50 \mathrm{MHz})$, high-resolution ultrasound (Vevo®; 2100 Imaging System, FUJIFILM VisualSonics, Toronto, Canada), taking the speed of sound in the tissue to be $1560 \mathrm{~m} / \mathrm{s}$. For each blood vessel and for each participating individual, three transverse sections and three longitudinal sections were acquired, all in the end-diastolic phase. To obtain one such image, a series of images were captured at 100 frames per second, and one image in the end-diastolic phase was identified through visual inspection. For one of the subjects, 15 images were acquired for each vessel and each view plane to provide sufficient data for comparing the mean and standard error of $h$ and $d$ produced by different users of the assessment tool. The Regional Ethical Review Board in Linköping (Sweden) approved the study protocol (Dnr 2011/451-31 and Dnr 2013/65-32), and informed consent was obtained from all subjects who were all healthy volunteers.

The resolution of the images is limited by the embedded noise. We evaluate this resolution by 
100 computing the intensity spectra of six acoustic images as follows. A two-dimensional discrete

101 Fourier transform (2DFT) is applied to the images. This 2DFT yields a complex matrix,

102 where each element $y_{i j}$ represents the amplitude and phase of a wave vector of wavelength $\lambda_{i j}$.

103 Here, $s_{i j}=\left|y_{i j}\right|^{2}$ is the variance contribution of this wave vector to the image. The intensity

104 spectrum of an image is the variance density as a function of wavelength, computed from the

105 histogram of the $\left(\lambda_{i j}, s_{i j}\right)$ data points. The resulting six intensity spectra are plotted with

106 arbitrary units for the intensity against wavelength $\lambda$ in Fig. $\underline{3}$. The common peak of these

107 spectra occur at $\lambda=37 \mu \mathrm{m}$, which we interpret as the resolution of the acoustic images.

109 There are three distinct echos appearing as light-colored patches in the acoustic images, as

110 described in the literature ${ }^{14}$ : Counting from the near wall toward the far, the first echo

111 originates from near wall blood-intima interface. This echo comes from the blood, so the

112 blood-intima interface is identified as the near boundary of this light patch. The second echo

113 originates from the far wall blood-intima interface, and comes from the vessel wall, so that

114 the blood-intima interface, again, is located at the near boundary of this patch. The third echo

115 originates from the far wall media-adventitia interface. This is visible by virtue of the fair

116 acoustic contrast between the dark-colored media and the lighter adventitia. Other echos in

117 the images are indistinct and less suitable for manual edge detection.

118 The six observers that perform measurements in this study are all employees with variable

119 previous experience of medical ultrasound. They prepared by reading an instruction, which

120 describes the measurement procedure as well as the origin of the ultrasonic echos from blood

121 vessels and how these represent blood-intima and media-adventitia interfaces. The objective

122 of the measurements is to evaluate the geometry of the blood vessel in terms of its wall

123 thickness $h$ and its nominal diameter $d$. To minimize measurement bias, information about 
124 which subject and which type of vessel, RA or CV, the ultrasonic image depicts is hidden

125 from the observers, the images are given randomized filenames, and their order of appearance

126 in the database is randomized.

127 For transverse sections (Fig. 1a), caliper measurements are performed as illustrated in

128 Fig. 1bcde. For the ellipse-fitting method, the observer provides one point set representing the 129 blood-intima interface, and one representing the media-adventitia interface of the blood 130 vessel (Fig. 1fgh) through the GUI. Two concentric ellipses are then fit in a least-squares 131 sense to these sets using a previously suggested method ${ }^{8}$. The fit is used for computing the 132 dimensions of the vessel ${ }^{15}$. This yields the average wall thickness $h_{\mathrm{e}}$ as well as the 133 circumference $C_{\mathrm{e}}$ of the elliptical mid-plane of the fitted ellipses ${ }^{16}$, and we take $h=h_{\mathrm{e}}$ and $d=$ $134 C_{\mathrm{e}} / \pi$.

135 For longitudinal sections, caliper measurements are performed as illustrated in Fig. $\underline{2}$ bcde.

136 For the polynomial fitting, different input techniques are used for the near and far wall. The

137 far wall has two distinct echos (Fig. 2a). The user provides two point sets for the far wall 138 sections (Fig. 2fg), and third-order polynomial functions are fit in a least-squares sense to 139 allow for undulations of the wall midplane, with a constant wall thickness ${ }^{15}$. The near wall 140 has only one distinct echo from the blood-intima interface. The user provides one point set 141 representing this interface, and a polynomial is fit (Fig. 2hi). The wall thickness of the near 142 wall is assumed to be the same as that of the far wall. The details of shape-fitting in a leastsquares sense using a previously suggested $\operatorname{method}^{8}$ is discussed at length in the electronic

144 supplement ${ }^{15}$. The nominal diameter is obtained as the average vertical distance between the 145 midplanes of the near and far walls. That is, the blood vessel is assumed to have a tubular 146 shape.

147 For each individual assessment, the type of blood vessel (RA/CV), the view plane 
148 (transverse/longitudinal), the measurement method (caliper/shape-fitting), the wall

149 thickness $h$, and the nominal diameter $d$ are stored. In addition, the observer judges whether

150 the image quality is sufficient to measure the diameter and wall thickness, respectively

151 (acceptable/uncertain/reject). Only data from assessments that were not rejected are included

152 in the subsequent analysis. Independent two-sample $t$-tests are conducted to detect significant

153 differences $(p<0.01)$ between results from different measurement methods or from different 154 observers.

\section{Results and Discussion}

156 The RAs from different subjects are denoted by $\mathrm{RA}_{i}, i=1, \ldots, 6$ where $i$ identifies the subject. Similarly, $\mathrm{CV}_{i}$ denotes the CVs of different subjects. Each one of these twelve blood vessels is evaluated using four different measurement methods: caliper measurement for

159 longitudinal sections, polynomial fit to longitudinal sections, caliper measurement for

160 transverse sections, and ellipse fit for transverse sections. The mean and standard deviation of the wall thickness and the nominal diameter of each methods and each blood vessel, $\mathrm{RA}_{i}$ and

$162 \mathrm{CV}_{i}$, are computed from the set of all images and all observers (Fig. $\underline{4}$ abcd).

163 The difference in mean wall thickness $h$ between different measurements methods is within

164 the variability of the measurements for both the RAs (Fig. $\underline{4 a}$ ) and the CVs (Fig. $\underline{4 b}$ ). Thus,

165 the measurement methods perform equally well when used to evaluate the wall thickness.

166 The standard deviation of the wall thickness (Fig. $\underline{4 a b)}$ is comparable to the resolution of the

167 images. It should be expected that lower frequency imaging systems, typically used in the 168 clinic, would give lower resolution.

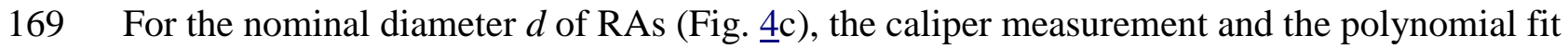
to longitudinal sections are in agreement within the variability of the measurements. The 
171 same is true in the case of CVs (Fig. 4 d). It can be concluded that the polynomial-fitting

172 measurements and caliper measurements yield the same level of precision. This implies that

173 marking the position of an interfaces with multiple data points and least-squares fitting a

174 shape is at least as precise as marking the position with a single point.

175 Caliper measurements for transverse sections give a much larger value for the nominal 176 diameter than caliper measurements for longitudinal sections, particularly for the CVs

177 (Fig. 4d) for which transverse sections give 39.5\% greater diameter on the average. Since the 178 typical causes for errors, e.g. misalignment of the view plane or compression of the vessel, is

179 linked to a reduction of the observed diameter rather than an increase, it is concluded that

180 transverse sections should be used for measuring the diameter of veins to reduce the 181 systematic error.

182 The greatest values for the nominal diameter are consistently reported using the ellipse-fitting

183 method for transverse sections (Fig. 4 cd). For the RAs, ellipse-fitting gives $4.5 \%$ greater

184 nominal diameter than the caliper measurements on transverse sections (Fig. $4 \mathrm{c}$ ) on the

185 average. For the CVs, ellipse-fitting gives $17.2 \%$ greater nominal diameter than the caliper

186 measurements on transverse sections (Fig. 4d). Since the ellipse-fitting takes the noncircular

187 shape of the transverse cross-section into account, we believe that ellipse-fitting enhances the

188 accuracy of this measurement. This is particularly important for veins since transverse

189 sections of veins deviate more from a circular geometry. An ellipse-fitting measurement of

190 the transverse section of a CV gives $62.7 \%$ greater nominal diameter than a caliper

191 measurement of the longitudinal section on the average.

192 We proceed to investigate the variation between different observers when assessing the same

193 blood vessel using ellipse-fitting for transverse (tr.) sections or caliper measurements for

194 longitudinal (lo.) sections (Fig. 5 $\underline{a}$ b). For this purpose, we consider the one subject, $i=5$, with 
19515 acquired images for each vessel type and view plane. For the wall thickness of $\mathrm{RA}_{5}$ and

$196 \mathrm{CV}_{5}$, and for each view plane, an independent two-sample $t$-test is conducted for each pair of

197 observers. This revealed significant differences $(p<0.01)$ between the observers' evaluations

198 in 19 out of $60 t$-tests. Significant differences $(p<0.01)$ between observers are found in 6 out

199 of 60 cases of the nominal diameter measurements. This demonstrates that different observers

200 judge the blood vessel geometry differently, particularly the wall thickness.

\section{Conclusions}

202 A new method for computer-aided assessment of the wall-thickness and nominal diameter of blood vessels from acoustic images was described and compared to caliper measurements.

204 The difference between wall thickness measurements using the caliper method and the shape-

205 fitting method is within the variability of the data. The standard deviation of the wall

206 thickness is comparable to the typical wavelength, $37 \mu \mathrm{m}$, of the noise. When considering the

207 nominal diameter, results deviate significantly between caliper and ellipse-fitting

208 measurements for transverse sections. We believe that this can be attributed to the

209 eccentricity of the cross-section, which is taken into account with the ellipse-fitting method.

210 It was demonstrated that different observers deliver significantly different results for the wall

211 thickness regardless of the method being used. This difficulty to interpret the echos in the

212 ultrasonic images is likely related to the noise-limited resolution, whose wavelength is in the

213 order of the variability of the measurements.

214 Our recommendation is that the nominal diameter should be measured using ultrasonic

215 images of the transverse section of small superficial vessels, and that the ellipse-fitting

216 assessment should be used to reduce systematic errors as much as possible. This is

217 particularly important for the $\mathrm{CV}$, where the effect of eccentricity can be as high as $63 \%$. 
218 The wall thickness measurements are less prone to systematic error, as no significant

219 difference can be demonstrated between longitudinal and transverse view planes. Considering

220 that caliper measurements are simple and well-established, we recommend that a caliper

221 measurement of either one of the view planes is used to assess the wall thickness.

222 Direction for future studies will be measurements in clinical situations where the vessel

223 diameter and wall thickness can be of interest. One such example would be to follow the

224 postoperative maturation of arteriovenous fistulas to be used for blood dialysis. Further

225 possible applications are stenosis in superficial vessels, and the rapid calcification processes

226 among patients with chronic kidney disease. In each case, an accurate, time-resolved

227 measurement of the vessel geometry contributes to the evaluation of development and

228 treatment. 
229 Acknowledgement

230 S.B.L. thanks the Swedish Research Council for financial support under contract Dnr: 621-

231 2012-3117. The Swedish Heart-Lung Foundation is acknowledged for financial support. 


\section{References}

233 1. Baek S, Rajagopal KR, and Humphrey JD. A theoretical model of enlarging intracranial

234 fusiform aneurysms. J Biomech Eng 2006; 128:142-149.

235 2. Valentín A and Humphrey JD. Evaluation of fundamental hypotheses underlying

236 constrained mixture models of arterial growth and remodelling. Philos T Roy Soc A 2009;

$237 \quad 367: 3585-3606$.

238 3. Satha G, Lindström SB, and Klarbring A. A goal function approach to remodeling of

239 arteries uncovers mechanisms for growth instability. Biomech Model Mechan 2014; 13:12432401259.

241 4. Lindström SB, Stålhand J, and Klarbring A. Modeling of the mechanobiological

242 adaptation in muscular arteries [published online ahead of print February 2017]. Eur J Mech

243 A-Solid. doi: 10.1016/j.euromechsol.2017.01.011.

244 5. Boron WF and Boulpaep EL. Medical physiology. Saunders; 2008.

245 6. Baek S, Valentín A, and Humphrey JD. Biomechanics of cerebral vasoplasm and its

246 resolution: II. Constitutive relations and model simulations. Ann Biomed Eng 2007; 35:1498-

2471509.

248 7. Fitzgibbon AW, Pilu M, and Fisher RB. Direct least-squares fitting of ellipses. IEEE

249 Trans Pattern Anal Mach Intell 1999; 21:476-480.

250 8. O’Leary P, Harker M, and Zsombor-Murray P. Direct and least square fitting of coupled

251 geometric objects for metric vision. IEEE Proc-Vis Image Signal Process 2005; 152:687252694 
253 9. Kluge K. Extracting road curvature and orientation from image edges points without 254 perceptual grouping into features. In Proc IEEE Intelligent Vehicles Symposium, Paris, 255 France: IEEE Press; 1994:109-114.

256 10. Khalifa OO, Khan IM, Assidiq AAM, Abdulla AH, and Khan S. A hyperbola-pair based 257 lane detection system for vehicle guidance. In Proc World Congress on Engineering and 258 Computer Science 2010 Vol I, San Fransisco, USA: IEEE Press; 2010:585-588.

259 11. Davies ER. Computer and Machine Vision: Theory, Algorithms, Practicalities.

260 Academic Press, 4th edition; 2012.

261 12. Malamas EN, Petrakis EGM, Zervakis M, Petit L, and Legat JD. A survey on industrial 262 vision systems, applications and tools. Image and Vision Computing 2003; 21:171-188.

263 13. Hansen DW and Ji Q. In the eye of the beholder: A survey of models for eyes and gaze. 264 IEEE Trans Pattern Anal Mach Intell 2010; 32:478-500.

265 14. Wendelhag I, Gustavsson T, Suurküla M, Berglund G, and Wikstrand L. Ultrasound 266 measurement of wall thickness in the carotid artery: Fundamental principles and description 267 of a computerized analysing system. Clin Physiol 1991; 11:565-577.

268 15. Supplement: Shape-fitting algorithms.

269 16. Bessel FW. The calculation of longitude and latitude from geodesic measurements 270 (1825). Astron Nachr 2010; 331:852-861. 

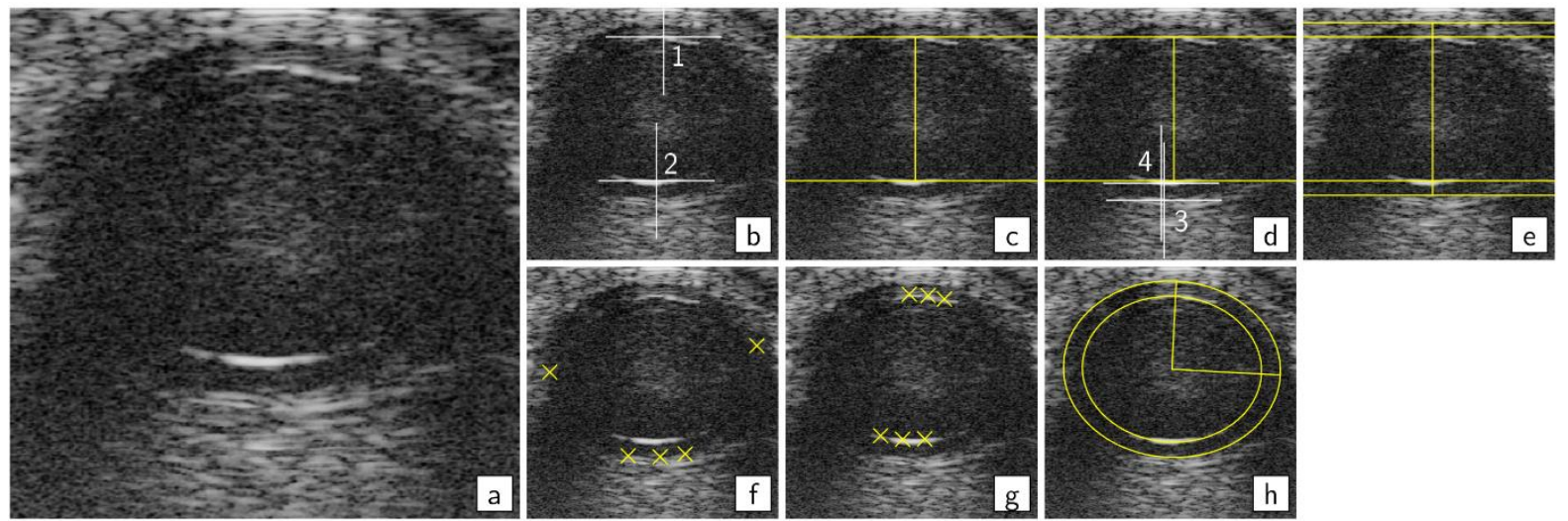

271

Figure 1: Assessment of (a) transverse section, near wall top, far wall bottom. (b-e) Caliper

272 measurement: the observer clicks opposite lumen boundary points 1 and 2, and the points

2733 and 4 on either side of the vessel wall. (f-h) Ellipse-fitting: the observer provides data

274 points for the fit by outlining the media-adventitia interface and then the blood-intima

275 interface. 


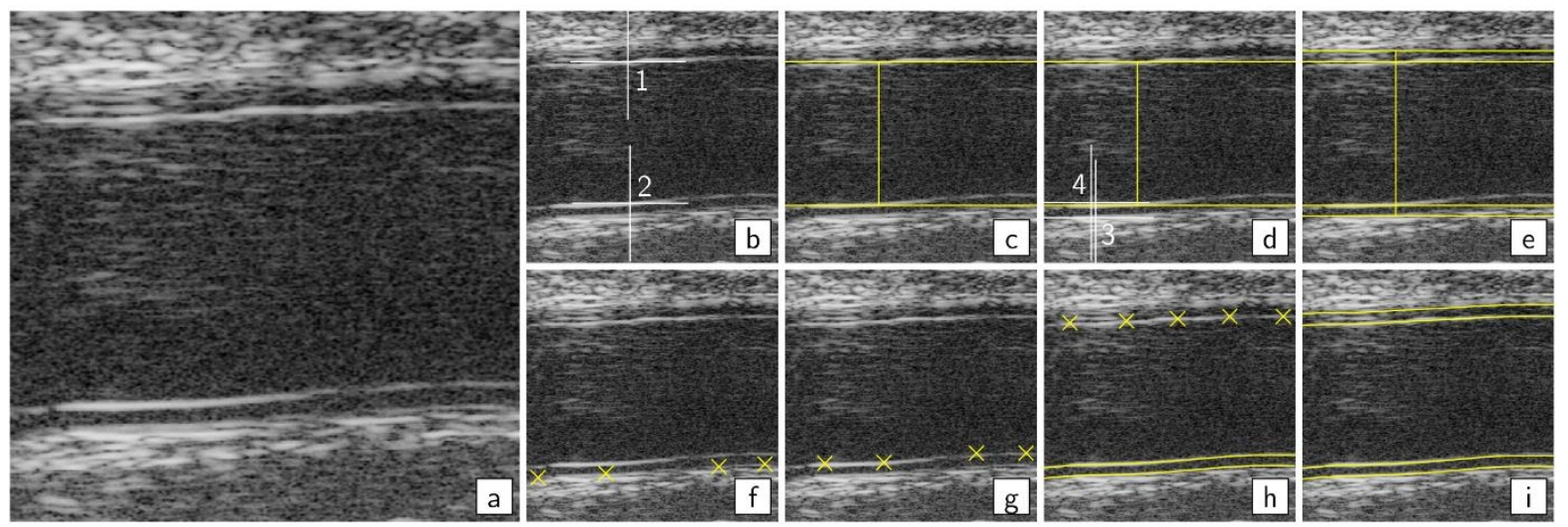

276 Figure 2: Assessment of (a) longitudinal section, near wall top, far wall bottom. (b-e) Caliper

277 measurement: the observer clicks opposite lumen boundary points 1 and 2, and the points

2783 and 4 on either side of the far vessel wall. (f-i) Polynomial-fitting: the observer provides

279 data points for the fit by outlining the media-adventitia interface of the far wall, the blood-

280 intima interface of the far wall, and the blood-intima interface of the near wall. 


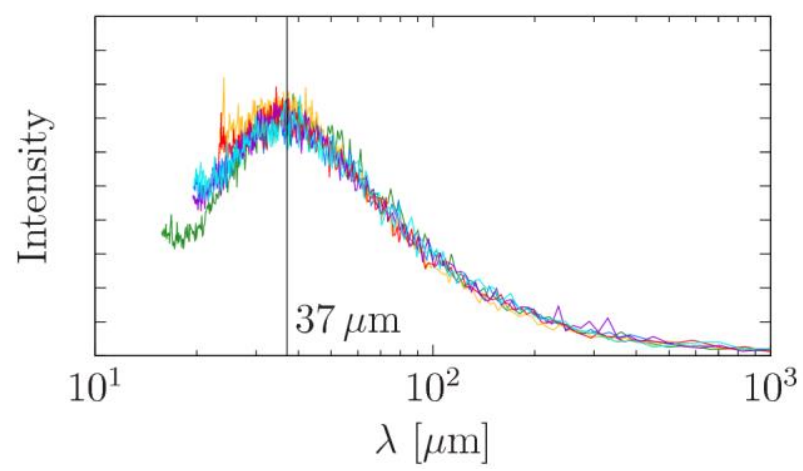

281 Figure 3: Normalized intensity of six acoustic images in arbitrary units as a function of the

282 wavelength. The peak at $37 \mu \mathrm{m}$ is the typical wavelength of the noise. 

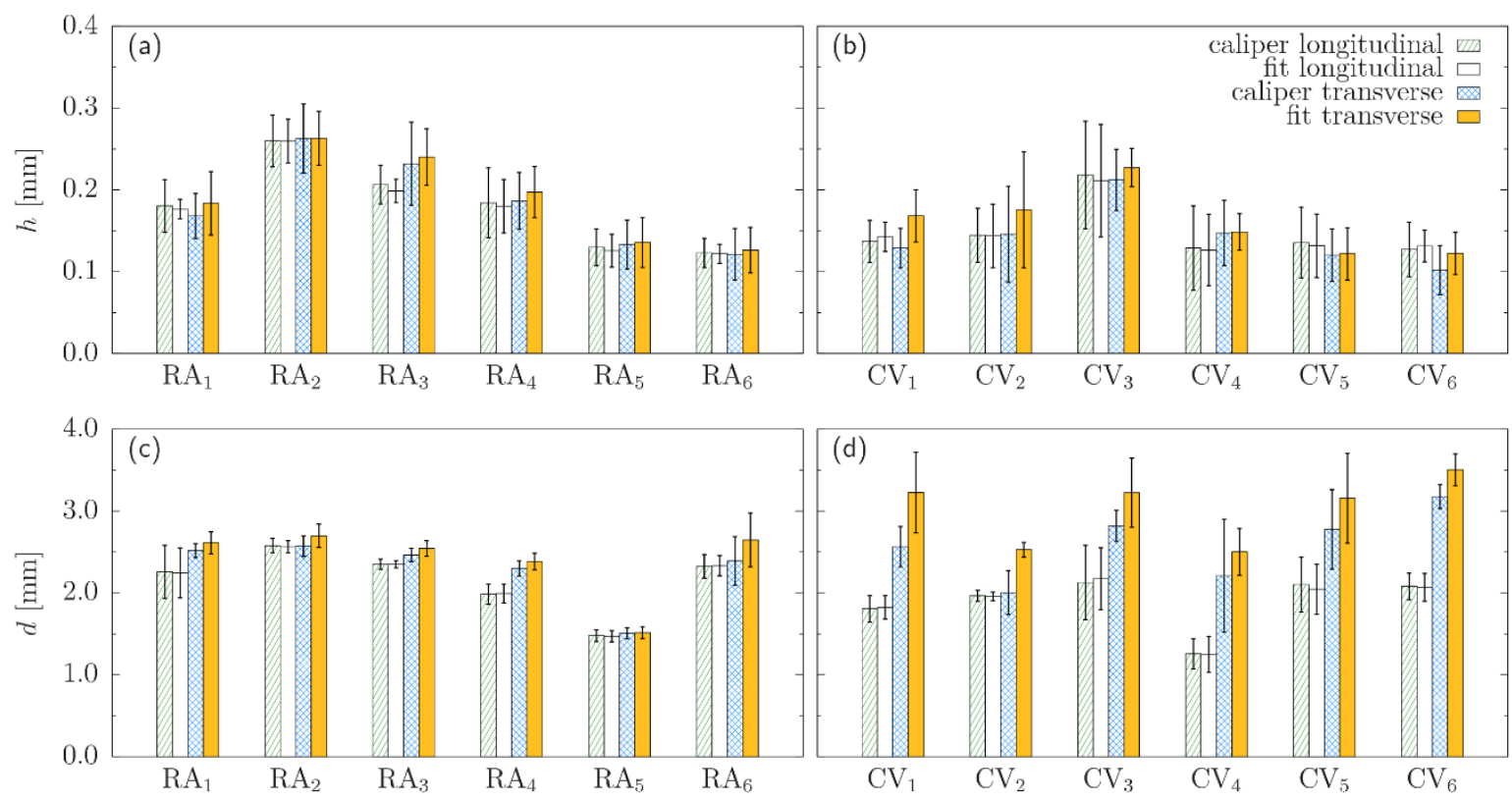

283 Figure 4: Wall thickness and nominal diameter measured using four different methods:

284 caliper measurement for longitudinal sections (slanted), polynomial fit to longitudinal

285 sections (white), caliper measurement for transverse sections (diamond), and ellipse fit for

286 transverse sections (solid). Each bar represents the mean of 16 measurements, and the error

287 bars represent the standard deviation. (a) Wall thickness of RAs. (b) Wall thickness of CVs.

(c) Nominal diameter of RAs. (d) Nominal diameter of CVs. 

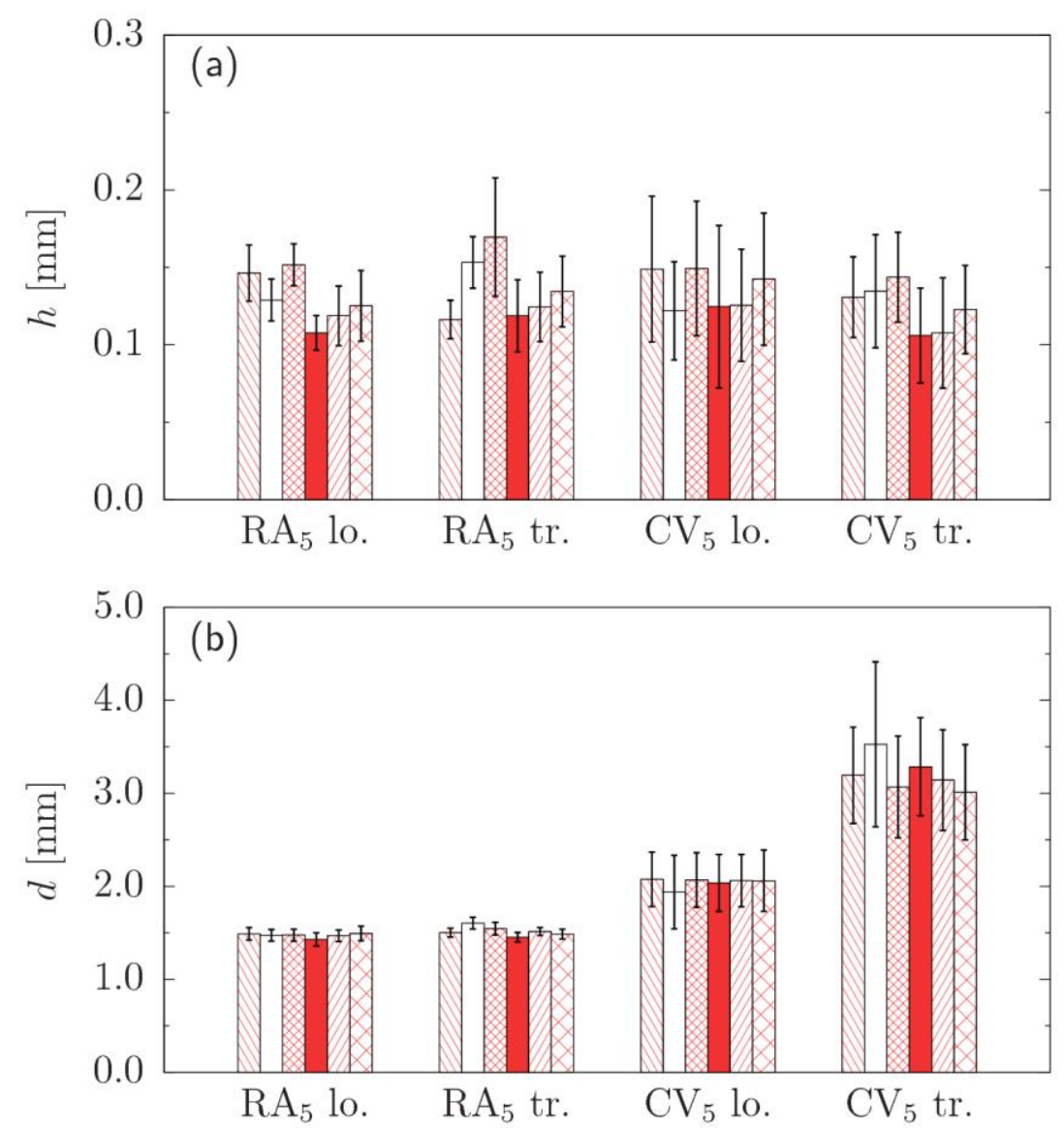

289 Figure 5: Comparison of measurements from different observers of the same vessel using

290 ellipse-fitting for transverse (tr.) sections or caliper measurements for longitudinal (lo.)

291 sections. Each bar represents the assessments of one observer of one vessel averaged over

292 multiple images. (a) Wall thickness. (b) Nominal diameter. 